Technical Report UTM 746, Matematica, University of Trento.

URL: http://eprints.biblio.unitn.it/

\title{
EXISTENCE AND STABILITY OF SQUARE-MEAN ALMOST PERIODIC SOLUTIONS TO A SPATIALLY EXTENDED NEURAL NETWORK WITH IMPULSIVE NOISE
}

\author{
STEFANO BONACCORSI, GIACOMO ZIGLIO
}

\begin{abstract}
Our work is concerned with a neural network with $n$ nodes, where the activity of the $k$-th cell depends on external, stochastic inputs as well as the coupling generated by the activity of the adjacent cells, transmitted through a diffusion process in the network. This paper aims to throw some light on time-varying, stochastically perturbed, neuronal networks. We show that when the coefficients oscillate around a reference value, with ascillations that are almost periodic and suitably small in percentage, then there exists a unique solution for the system, that is almost periodic and uniformly bounded in the mean square norm for all times.
\end{abstract}

\section{INTRODUCTION}

Cellular neural networks have attracted a large amount of mathematical research due to their applications in various modelling models. These applications depend (or, on the other hand, influence) the shape and dynamics of the network. Neural networks should have a spatial extent, that is usually negletted in the current mathematical research. Our model is related to neural networks 12 and cellular neural networks [6, 7], although their dynamics is usually modelled through a finite dimensional system (possibly using some delay to model the physical extension of transmitters).

Since their introduction, such models have been used in several applications (from image processing to pattern recognition and other areas); many authors have analyzed the dynamical behavior of such systems with particular respect to stability, periodicity and almost periodicity of the system (compare for instance [5]). In general, neural networks are complex and large-scale nonlinear dynamical systems. To the best of our knowledge, few authors have considered periodic oscillatory solutions for large-scale networks with stochastic impulses. Also literature dealing with time networks parameters appears to be scanty. Such studies, however, are very important to understand the dynamics of neural networks in time-varying environments.

Let us briefly introduce our model, following the approach of [3, 4, Let $G=$ $(V, E)$ be a graph with $n$ vertices and $m$ edges; to each node $\mathrm{v}_{i} \in V$ is associated a

2000 Mathematics Subject Classification. 37N25, 34K14, $60 \mathrm{H} 15$.

Key words and phrases. diffusion equations on networks, stochastic impulses, almost periodic solutions.

The research of G. Ziglio was supported by Project NeSt funded by Provincia Autonoma di Trento (P.A.T.) within Bando unità di ricerca 2006. 
variable $p_{i}(t)$ evolving according to the stochastic equation

$$
\frac{\partial}{\partial t} p_{i}(t)=-b_{i}(t) p_{i}(t)+k_{i}(t)+\sigma_{i}\left(t, p_{i}(t)\right) \frac{\partial}{\partial t} L\left(t, \mathrm{v}_{i}\right),
$$

for all $t \in \mathbb{R}$ and $i=1, \ldots, n$. The coefficients $b_{i}(t)>0$ represent the passive decay rate of the cell activity at node $v_{i}$. In words, we may say that the variation of the value of $p_{i}(t)$ depends on the feedback from the network $k_{i}(t)$ and a stochastic input, which takes into account both the intrinsic cellular noise and the external input signals. All the weights $b_{i}$ and $\sigma_{i}$ and the inputs $k_{i}$ should be almost periodically varying in time. Biological motivations, going back at least to the works of Kallianpur [13, lead us to model this term by a Lévy-type process (compare Section 2.3 for details). $L\left(t, \mathrm{v}_{i}\right), i=1, \ldots, n$, represent the stochastic perturbation acting on each node, due to the external surrounding, and $\frac{\partial}{\partial t} L\left(t, v_{i}\right)$ is the formal time derivative of the process $L$, which takes a meaning only in integral sense.

A different feature of this paper with respect to the existing literature on cellular neural networks is that we take into account that the network have a spatial extension and that each signal is propagated along the network with a diffusive mechanism. To be precise, we assume that each edge $e_{j}$ in the network is isomorphic to the segment $[0,1]$. The electrical potential in the network shall be denoted by $\bar{u}(t, x)$ where $\bar{u} \in\left(L^{2}(0,1)\right)^{m}$ is the vector $\left(u_{1}(t, x), \ldots, u_{m}(t, x)\right)$. We impose a general diffusion equation on every edge with time-dependent, almost periodic coefficients:

$$
\frac{\partial}{\partial t} u_{j}(t, x)=\frac{\partial^{2}}{\partial x^{2}} u_{j}(t, x)-v_{j}(t) u_{j}(t, x)+f_{j}\left(t, x, u_{j}(t, x)\right),
$$

for all $(t, x) \in \mathbb{R} \times(0,1)$ and all $j=1, \ldots, m$.

Then, we assume that the feedback from the network is given by a Kirchhoff's type function of the electric potential on the edges:

$$
k_{i}(t)=\left(1+c_{i}(t)\right) \sum_{j=1}^{m} \phi_{i j} u_{j}^{\prime}\left(t, \mathrm{v}_{i}\right)
$$

where the sum is taken over all edges $\mathrm{e}_{j}$ that insist on the node $\mathrm{v}_{i}$ and $\phi_{i j}$ are coefficients that depend on the geometry of the network. The functions $c_{i}(t)$ measure the oscillations around the mean value of the influence of the network on the evolution in every node.

In this paper we consider a situation where the parameters of the system are not constant in time but can vary, almost periodically, around the mean value. Such variation is supposed to be small in percentage with respect to the mean value itself, see Hypothesis 2.2 for a precise statement.

In case of constant coefficients, the dynamics of the linearized system is described by an evolution semigroup $e^{t \mathcal{A}}$ on a suitable product space $\mathcal{H}$; properties of this semigroup are given in [3, 4. In case of time-varying coefficients, the same system is described by a family of evolution operators $\mathcal{U}(t, s)$ generated by the non-autonomous perturbation $\mathcal{A}(t)=\mathcal{A}+\mathcal{V}(t)$.

Almost periodicity for stochastic differential equations in infinite dimensional spaces has been studied by many authors, compare for instance [9, 17. Let $H$ denote a real separable Hilbert space. We recall that a continuous function $f$ : $\mathbb{R} \rightarrow H$ is almost periodic if for every $\varepsilon>0$ there exists a number $\ell(\varepsilon)>0$ such that each interval $(a, a+\ell(\varepsilon)), a \in \mathbb{R}$, contains an almost period $\tau=\tau_{\varepsilon}$ and the 
estimate $\|f(t+\tau)-f(t)\| \leq \varepsilon$ holds for all $t \in \mathbb{R}$. The space of almost periodic $H$-valued functions will be denoted by $A P(\mathbb{R} ; H))$. We shall also mention that any almost periodic function is bounded and uniformly continuous and that the space of all almost periodic functions from $\mathbb{R}$ to $H$, which we denote by $A P(\mathbb{R}, H)$ is a complex Banach space in the canonical way.

It is worth noticing that, when translated in an abstract setting, our problem takes the form of an evolution problem on an Hilbert space $\mathcal{H}$ with stochastic multiplicative term that is driven by a (finite dimensional, two-sided) Lévy process, having the form

$$
\mathrm{d} X(t)=[\mathcal{A}(t) X(t)+F(t, X(t))] \mathrm{d} t+G(t, X(t)) \mathrm{d} L(t), \quad t \in \mathbb{R} .
$$

The precise assumptions on the coefficients in (1.1) and 1.2 will be given in Section 2. There we also show that $(\mathcal{A}(t), D(\mathcal{A}))$ for $t \in \mathbb{R}$ generates an evolution family $\{\mathcal{U}(t, s): t \geq s, t, s \in \mathbb{R}\}$.

Next, we introduce the concept of mild solution for the problem (1.3).

Definition 1.1. An $\mathcal{H}$-valued predictable process $X(t)$ is said to be a mild solution of (1.3) if $\mathbb{P}$-a.s.

$$
X(t)=\mathcal{U}(t, s) X(s)+\int_{s}^{t} \mathcal{U}(t, r) F(r, X(r)) \mathrm{d} r+\int_{s}^{t} \mathcal{U}(t, r) G(r, X(r)) \mathrm{d} L(r)
$$

for all $t \geq s, t, s \in \mathbb{R}$.

The following definition introduces the concept of almost periodicity with regard to (Hilbert space valued) square integrable stochastic process. This concept has been applied to study the existence and uniqueness of square-mean almost periodic mild solutions to different classes of non-autonomous semilinear stochastic differential equations driven by two-sided Wiener processes (see for instance Bezandry and Diagana [1, 2]).

Definition 1.2. Let $X: \mathbb{R} \rightarrow L^{2}(\Omega ; \mathcal{H})$ be a mean-square continuous stochastic process. Then $X$ is said to be square-mean almost periodic on $\mathbb{R}$ if, for each $\varepsilon>0$, the set

$$
T(X, \varepsilon)=\left\{\tau \mid \mathbb{E}\|X(t+\tau)-X(t)\|^{2} \leq \varepsilon, \quad \forall t \in \mathbb{R}\right\}
$$

is relatively dense (see e.g. [10]); equivalently (see e.g. [2]) $X$ is square-mean almost-periodic if, for all $\varepsilon>0$, it is possible to find a real number $\ell=\ell(\varepsilon)>0$ such that for any interval of length $\ell(\varepsilon)$, there exists a number $\tau=\tau_{\varepsilon}$ in this interval with

$$
\sup _{t \in \mathbb{R}} \mathbb{E}\|X(t+\tau)-X(t)\|^{2}<\varepsilon .
$$

In the next lemma we collect some properties of square-mean almost periodic processes (compare for instance [1, §2.]).

Lemma 1.3. A square-mean almost periodic process $X \in A P\left(\mathbb{R} ; L^{2}(\Omega ; \mathcal{H})\right)$ satisfies

(i) the mapping $t \rightarrow \mathbb{E}\|X(t)\|^{2}$ is uniformly continuous;

(ii) there exists a constant $N>0$ such that $\sup _{t \in \mathbb{R}} \mathbb{E}\|X(t)\|^{2} \leq N$. 
We can now proceed to present our main result. The following theorem holds provided it is verified a smallness condition on the Lipschitz constants of the coefficients $F$ and $G$, see $(3.2)$ for a precise statement.

Theorem 1.4. In our assumptions, there exists an unique square-mean almost periodic mild solution $X(t)$ of (1.3) which is the solution of the following integral equation

$$
X(t)=\int_{-\infty}^{t} \mathcal{U}(t, r) F(r, X(r)) \mathrm{d} r+\int_{-\infty}^{t} \mathcal{U}(t, r) G(r, X(r)) \mathrm{d} L(r) \quad \text { for each } t \in \mathbb{R} .
$$

\section{AbStract SETting FOR The NeURAL NETWORK MODEL}

Let $\bar{u}$ denote the electric potential on the whole dendritic network, including the ramification points (synapses or dendritic junctions or somata). $\bar{u}=\bar{u}(t, x)$ is a function of the position $x$ along the network and of time $t$ only and $\bar{u}(t, x)$ denotes the deviation from resting potential, which we rescale to 0 for simplicity (in concrete neurophysical measurements it is of approx. $-70 \mathrm{mV}$ ). The reference space for $\bar{u}$ is the Hilbert space $\mathbb{H}=\left(L^{2}(0,1)\right)^{m}$. The choice to take real-valued Hilbert spaces is done for coherence with the physical problem at hand; no further mathematical complications arise if we had taken into account complex-valued functions instead.

As already mentioned in the introduction, up to considering suitable rescaling diffusion parameters in the equations, we may and do parametrize the edges as intervals of unitary length. The diffusion of electric potential along every edge of the graph thus follows the equation (1.2). We avoid to introduce more general network elliptic operators: in fact, under uniform ellipticity assumptions this case is known to present no serious mathematical challenges over the basic case of a plain network Laplacian: compare [16].

On the Sobolev space $\mathbb{H}^{2}=\left(H^{2}(0,1)\right)^{m}$ we introduce the operator-valued matrix

$$
A \bar{u}=\left(\begin{array}{lll}
\frac{\partial^{2}}{\partial x^{2}} u_{1} & \cdots & \frac{\partial^{2}}{\partial x^{2}} u_{m}
\end{array}\right)^{\top}
$$

with maximal domain $D(A)=\mathbb{H}^{2}$. With the aid of this operator, we may write the linear, autonomous part of 1.2 in the form $\frac{d}{d t} \bar{u}(t)=A \bar{u}$.

We assume that somata and synapses are equipotential; if $q_{i}(t)$ is the electric potential in the junction node $\mathrm{v}_{i}$ at time $t$, then it shall hold $q_{i}(t)=u_{j}\left(t, \mathrm{v}_{i}\right)$ for every edge $\mathrm{e}_{j}$ that insists on the node $\mathrm{v}_{i}$.

The potential $q_{i}(t)=\bar{u}\left(t, \mathrm{v}_{i}\right)$ undergoes internal dynamics, subject to internal electrical activity and a stochastic feedback from the dendritic network. In the spirit of Rall's lumped soma model (see e.g. 14]) we impose general, possibly absorbing (and also possibly non-local) nodal conditions.

Remark 2.1. We recall some useful notation from graph theory. For every $i=$ $1, \ldots, n$, we let $\Gamma\left(\mathrm{v}_{i}\right)=\left\{\mathrm{e}_{j}: \mathrm{v}_{i}=\mathrm{e}_{j}(0)\right.$ or $\left.\mathrm{e}_{j}(1)\right\}$. Also, the incidence matrix $\Phi$ of the graph, $\Phi=\Phi^{+}-\Phi^{-}$, is defined in terms of the incoming and outgoing incidence matrices defined by

$$
\phi_{i j}^{+}:=\left\{\begin{array}{ll}
1 & \text { if } \mathrm{e}_{j}(0)=\mathrm{v}_{i}, \\
0 & \text { otherwise, }
\end{array} \quad \text { and } \quad \phi_{i j}^{-}:= \begin{cases}1 & \text { if } \mathrm{e}_{j}(1)=\mathrm{v}_{i}, \\
0 & \text { otherwise, }\end{cases}\right.
$$

respectively. 
For the sake of notational simplicity, let us introduce the Kirchhoff operator K mapping the Sobolev space $\mathbb{H}^{2}$ of vector-valued twice weakly differentiable functions into the boundary space $\mathbb{R}^{n}$, defined by

$$
K \bar{u}:=\left(\begin{array}{c}
\sum_{\mathrm{e}_{j} \in \Gamma\left(\mathrm{v}_{1}\right)} \phi_{1 j} u_{j}^{\prime}\left(t, \mathrm{v}_{1}\right) \\
\vdots \\
\sum_{\mathrm{e}_{j} \in \Gamma\left(\mathrm{v}_{n}\right)} \phi_{n j} u_{j}^{\prime}\left(t, \mathrm{v}_{n}\right)
\end{array}\right) .
$$

Thus, the vector $K \bar{u}$ represents the differences between incoming and outgoing flows in each of the nodes of the network. The presence of small perturbations in the system is described by the additional term

$$
K(t) \bar{u}:=\left(\begin{array}{cc}
c_{1}(t) & \sum_{\mathrm{e}_{j} \in \Gamma\left(\mathrm{v}_{1}\right)} \phi_{1 j} u_{j}^{\prime}\left(t, \mathrm{v}_{1}\right) \\
& \vdots \\
c_{n}(t) & \sum_{\mathrm{e}_{j} \in \Gamma\left(\mathrm{v}_{1}\right)} \phi_{n j} u_{j}^{\prime}\left(t, \mathrm{v}_{n}\right)
\end{array}\right)
$$

where the coefficients $c_{i}(t)$ are continuous functions.

We assume that $b_{i}(t)=b_{i}\left(1+\delta_{i}^{b}(t)\right)$ is a time dependent function that oscillates around a given value $b_{i}$, which defines the inhibitory properties of each node. On the boundary space $\mathbb{R}^{n}$ we introduce the matrix

$$
B+B(t)=\operatorname{diag}\left(b_{1}, \ldots, b_{n}\right)+\operatorname{diag}\left(b_{1} \delta_{1}^{b}(t), \ldots, b_{n} \delta_{n}^{b}(t)\right) .
$$

It shall be noticed that in [3], more general, possibly nonlocal, forms of the matrix $B$ are considered.

Also, in order to model the fluctuations of the diffusion operator, we introduce the matrix-valued operator

$$
\left.V(t)=\operatorname{diag}\left(v_{1}(t), \ldots, v_{m}(t)\right)\right) .
$$

With the above notation, problem $(1.1)-(1.2)$ can be written as an abstract nonautonomous Cauchy problem on the product space $\mathcal{H}=\mathbb{H} \times \mathbb{R}^{n}$ endowed with the natural inner product

$$
\langle X, Y\rangle_{\mathcal{H}}=\langle\bar{u}, \bar{v}\rangle_{\mathbb{H}}+\langle p, q\rangle_{\mathbb{R}^{n}}, \quad \text { where } X=\left(\begin{array}{c}
\bar{u} \\
p
\end{array}\right), Y=\left(\begin{array}{c}
\bar{v} \\
q
\end{array}\right) \in \mathcal{H}
$$

We introduce the family of linear matrix operators $\mathcal{A}(t)$ for $t \in \mathbb{R}$ on the space $\mathcal{H}$, given in the form

$$
\mathcal{A}(t)=\mathcal{A}+\mathcal{V}(t):=\left(\begin{array}{cc}
A & 0 \\
K & -B
\end{array}\right)+\left(\begin{array}{cc}
V(t) & 0 \\
K(t) & -B(t)
\end{array}\right)
$$

with domain

$$
D(\mathcal{A}(t))=D(\mathcal{A})=\left\{X=(\bar{u}, p) \in \mathcal{H}: \bar{u} \in D(A), u_{j}\left(\mathrm{v}_{i}\right)=p_{i} \text { for every } j \in \Gamma\left(\mathrm{v}_{i}\right)\right\} .
$$

In next section, we consider the Cauchy problem

$$
\left\{\begin{array}{l}
\dot{X}(t)=\mathcal{A}(t) X(t), \\
X(0)=X_{0}
\end{array}\right.
$$

where $u_{0}=\left(u_{j}(0, \cdot)\right)_{j=1, \ldots, m} \in C([0,1])^{m}$ is the $\mathbb{H}$-valued vector of initial conditions, $p_{0}=\left(u\left(0, v_{i}\right)\right)_{i=1, \ldots, n}$ and $X_{0}=\left(u_{0}, p_{0}\right)$ is the initial condition in 2.2. We 
obtain that the operator family $\mathcal{A}(t)$ generates an evolution family of operators with suitable properties that are related to the almost periodic behaviour of the coefficients.

2.1. Generation of an evolution family of operators. We recall from [4, Proposition 2.4] that the operator $\mathcal{A}$ is self-adjoint, dissipative and has compact resolvent; therefore, the semigroup $\{\mathcal{T}(t), t \geq 0\}$ generated by $\mathcal{A}$ is strongly continuous, analytic, contractive and exponentially bounded, with growth bound $\omega_{0}$ given by the strictly negative spectral bound of the operator $\mathcal{A}$.

Using the above stated properties of $\mathcal{A}$, we can introduce the interpolation spaces $\mathcal{H}^{2 s}=(\mathcal{H}, D(\mathcal{A}))_{s, 2}$ for every $s \in[0,1]$.

As we mentioned in the introduction, we are interested in the case of small, almost periodic perturbations of the mean value. We suppose that such perturbations do not overcome a fixed percentage $\delta<1$ of the mean value; we state this condition in the following assumption.

Hypothesis 2.2. The functions $v_{j}(t): \mathbb{R}_{+} \rightarrow \mathbb{R}, \delta_{i}^{b}(t): \mathbb{R}_{+} \rightarrow \mathbb{R}$ and $c_{i}(t): \mathbb{R}_{+} \rightarrow$ $\mathbb{R}$, for $i=1, \ldots, n$ and $j=1, \ldots, m$ are continuous and bounded and locally Hölder continuous. Moreover, there exists a constant $0<\delta<1$ such that

$$
\left|v_{j}(t)\right| \leq \delta, \quad\left|\delta_{i}^{b}(t)\right| \leq \delta, \quad\left|c_{i}(t)\right| \leq \delta .
$$

Under previous assumption, the operator $\mathcal{V}(t): \mathbb{R}_{+} \rightarrow L\left(\mathcal{H}^{\alpha}, \mathcal{H}\right)$ is locally Hölder continuous for every $\alpha>\frac{3}{2}$ and uniformly bounded: there exists a constant $C=C_{\alpha}$ such that the following estimate holds:

$$
\|\mathcal{V}(t)\|_{L\left(\mathcal{H}^{\alpha}, \mathcal{H}\right)} \leq C_{\alpha} \delta
$$

Then it follows from [11, Section 7.1] that there exists a family of evolution operators $U(t, s)$ associated to $\mathcal{A}(t)=\mathcal{A}+\mathcal{V}(t)$ and

$$
\|U(t, s)\| \leq M_{1} e^{\omega_{V}(t-s)}
$$

with

$$
\omega_{V}=\omega_{0}+\left(C_{\alpha} \delta\right)^{1 /(1-\alpha)} .
$$

Remark 2.3. If the constant $\delta$ in Hypothesis 2.2 is taken suitably small, then it is possible to take $\omega_{V}<0$.

Lemma 2.4. The operators $\mathcal{V}(t)$ are almost-periodic in the space $\mathcal{L}(D(\mathcal{A}), \mathcal{H})$.

Proof. This is a consequence of the fact that $A P(\mathbb{R})$ is an algebra and it is closed under the lattice operations on real functions, see [10, Theorem 1.9]. Therefore, the maximum of a finite number of almost periodic functions is an almost periodic function. In our framework, this maximum coincides with the operator norm $\|\mathcal{V}(t)\|_{\mathcal{L}(D(\mathcal{A}), \mathcal{H})}$ and the thesis is proved.

2.2. The nonlinear coefficients. We conclude our construction with the nonlinear coefficients $F$ and $G$.

In the definition of the diffusion process along every edge $\mathrm{e}_{j}$, see Eq. 1.2 , the function $f_{j}(t, x, u)$ takes real values and it is defined in the set $\mathbb{R} \times(0,1) \times \mathbb{R}$. 
Hypothesis 2.5. For every $j \in\{1, \ldots, m\}$, the map $f_{j}: \mathbb{R} \times(0,1) \times \mathbb{R} \rightarrow \mathbb{R}$ is continuous and mean square almost periodic, uniformly with respect to $u \in \mathbb{R}$.

Further, the function $f_{j}(t, x, \cdot): \mathbb{R} \rightarrow \mathbb{R}$ is Lipschitz continuous uniformly in $(t, x) \in \mathbb{R} \times(0,1)$.

Remark 2.6. An example of interest for us, in the context of Eq. 1.2), is the McKean's caricature to the cubic $h(u)=H(u-a)-u$, for some Hölder continuous and almost periodic function $0<a(t, x)<1$. The linear part $-u$ can be incorporated into the definition of the operator $\mathcal{A}$, thus leaving us concerned with the Heaviside function. We take $f(t, x, u)$ to be a smooth approximation of $H(u-a)$ so that $f(t, u)$ enters in our framework.

Setting $f(t, \bar{u}): \mathbb{R} \times \mathbb{H} \rightarrow \mathbb{H}$ by $f(t, \bar{u})=\left(f_{1}\left(t, u_{1}\right), \ldots, f_{m}\left(t, u_{m}\right)\right)$, we further define

$$
F(t, X)=\left(\begin{array}{c}
-f(t, \bar{u}) \\
0
\end{array}\right) \quad \text { for } X=\left(\begin{array}{c}
\bar{u} \\
p
\end{array}\right) .
$$

Next we turn to the diffusion coefficient $G$. We require the following.

Hypothesis 2.7. For every $i \in\{1, \ldots, n\}$, the map $\sigma_{i}: \mathbb{R} \times \mathbb{R} \rightarrow \mathbb{R}$ is continuous and almost periodic, uniformly with respect to $u \in \mathbb{R}$.

Further, the function $\sigma_{i}(t, \cdot): \mathbb{R} \rightarrow \mathbb{R}$ is Lipschitz continuous uniformly in $t \in \mathbb{R}$.

We set

$$
G(t, X)\left(\begin{array}{c}
\bar{v} \\
q
\end{array}\right)=\left(\begin{array}{c}
0 \\
\left(\sigma_{1}\left(t, p_{1}\right) q_{1}, \ldots, \sigma_{n}\left(t, p_{n}\right) q_{n}\right)^{\top}
\end{array}\right) \quad \text { for } X=\left(\begin{array}{c}
\bar{u} \\
p
\end{array}\right)
$$

where $\left(\begin{array}{l}\bar{v} \\ q\end{array}\right) \in \mathcal{H}$. In our assumptions, there exist constants $K_{F}$ and $K_{G}$ such that for all $X, Y \in L^{2}(\Omega ; \mathcal{H})$

$$
\begin{aligned}
\mathbb{E}\|F(t, X)-F(t, Y)\|_{\mathcal{H}}^{2} & \leq K_{F} \mathbb{E}\|X-Y\|^{2} \\
\mathbb{E}\|G(t, X)-G(t, Y)\|_{\mathcal{L}(\mathcal{H})}^{2} & \leq K_{G} \mathbb{E}\|X-Y\|^{2}
\end{aligned}
$$

uniformly in $t \in \mathbb{R}$.

Remark 2.8. We conclude this section with a simple, but crucial, observation that we eill use several times in next section: compare, for instance 8 , Proposition 3.21]. Given a finite family $\left\{\phi_{1}, \ldots, \phi_{k}\right\}$ of almost periodic mappings, it follows that for every $\varepsilon$ there exist common $\epsilon$-translation numbers for these functions and, in particular, the vector valued function $\phi=\left(\phi_{1}, \ldots, \phi_{k}\right)$ is almost periodic.

2.3. Noise. All stochastic elements are defined on a filtered probability space $\left(\Omega, \mathcal{F},\left\{\mathcal{F}_{t}\right\}, \mathbb{P}\right)$ satisfying the usual hypotheses of right continuity and completeness. For the sake of simplicity we assume that random disturbances occour only in the nodes of the graph; the extension to a stochastic version of the diffusion equation 1.2 can be handled with essentially the same techniques and leads to similar results. We shall consider an $n$-dimensional Lévy process $\left\{L(t), t \in \mathbb{R}_{+}\right\}$, i.e., a stochastically continuous, adapted process, with $L(0)=0$ almost surely, stationary and independent increments and càdlàg trajectories, hence with discontinuities of jump type. By the classical Lévy-Itô decomposition theorem, the Lévy process $L(t)$ 
has a decomposition

$$
L(t)=m t+Q W(t)+\int_{\|x\| \leq 1} x[N(t, \mathrm{~d} x)-t \nu(\mathrm{d} x)]+\int_{\|x\|>1} x N(t, \mathrm{~d} x), \quad t \geq 0
$$

where $m \in \mathbb{R}^{n}, Q$ is a symmetric nonnegative operator, $\left\{W(t), t \in \mathbb{R}^{+}\right\}$is a cylindrical Brownian motion and the Lévy measure $\nu(\mathrm{d} x)$ is $\sigma$-finite on $\mathbb{R}^{n} \backslash\{0\}$ and such that $\int \min \left(1, x^{2}\right) \nu(\mathrm{d} x)<\infty$. We denote by $\tilde{N}(\mathrm{~d} t, \mathrm{~d} x):=N(\mathrm{~d} t, \mathrm{~d} x)-\mathrm{d} t \nu(\mathrm{d} x)$ the compensated Poisson measure.

In (1.3) we consider a two-sided Lévy process obtained as follows:

$$
L(t):= \begin{cases}L(t), & t \geq 0, \\ L^{\prime}(-t), & t<0,\end{cases}
$$

with $L^{\prime}$ an independent copy of $L$, on the naturally associated filtration $\left\{\tilde{\mathcal{F}}_{t}, t \in \mathbb{R}\right\}$.

Below we state the precise form of the noise that we consider in this paper. Note however that combining the results in [2] with ours and appealing to the Lévy-Itô decomposition theorem (2.6), one could rather easily obtain corresponding results for evolution equations driven by general square-integrable Lévy noise.

Hypothesis 2.9. We assume that

$$
\int_{\|x\|>1} x \nu(\mathrm{d} x)=0 .
$$

We also assume throughout that the Lévy process is a pure jump process, i.e. $m \equiv 0$ and $Q \equiv 0$.

Therefore, the stochastic term is driven by an impulsive noise of the form

$$
L(t)=\int_{H} x \tilde{N}(t, \mathrm{~d} x) .
$$

We suppose that the measure $\nu$ has finite second order moment, i.e.

$$
\int_{H}\|x\|^{2} \nu(\mathrm{d} x)=: \nu_{2}<\infty .
$$

In particular, condition 2.9 implies that the generalized compound Poisson process $\int_{\|x\|>1} x N(t, \mathrm{~d} x)$ has finite moments of first and second order.

\section{MAin RESUlt}

In this section we discuss the existence of an almost-periodic solution for the abstract equation (1.3). Our main result, Theorem 3.4 below, holds in a fairly general setting that comprises, but is not confined to the framework of a stochastic equation on a network. Our presentation aims to maintain this generality without losing sight of the problem at hand.

$(\mathrm{A}: 1)\{\mathcal{A}(t), t \in \mathbb{R}\}$ is a family of linear operators such that

(a) for any $t \in \mathbb{R}$, the operator $\mathcal{A}(t)$ generates an analytic semigroup $\left\{e^{\tau \mathcal{A}(t)}\right.$ $\tau \geq 0\}$

(b) there exists a unique strongly continuous evolution family $\{\mathcal{U}(t, s)\}$ such that $u(t)=\mathcal{U}(t, s) x_{0}$ is the unique classical solution of $u^{\prime}(t)=$ $\mathcal{A}(t) u(t)$ with initial condition $u(s)=x_{0}$, for every $x_{0} \in \mathcal{H}$ and $s \leq t$; 
(c) $\{\mathcal{U}(t, s)\}$ is continuous in $\mathcal{L}(\mathcal{H})$ for $s<t \in \mathbb{R}$ and there exists $M_{1} \geq 1$ and $\omega_{V}<0$ such that $\|\mathcal{U}(t, s)\| \leq M_{1} e^{\omega_{V}(t-s)}$ for all $t \geq s \in \mathbb{R}$.

Notice that assumption (A:1) holds in our framework as a consequence of [3, Proposition 2.4] and the results in Section 2.1 above.

By previous assumption it follows that $\Sigma_{\phi} \cup\{0\} \subset \rho\left(\mathcal{A}(t)-\omega_{0}\right)$ and there exists a constant $K>0$ such that

$$
\left\|R\left(\lambda, \mathcal{A}(t)-\omega_{0}\right)\right\|_{\mathcal{L}(\mathcal{H})} \leq \frac{K}{1+|\lambda|}
$$

for every $\Re \lambda>\omega_{0}, t \in \mathbb{R}$ and $\lambda \in \Sigma_{\phi}=\{\lambda \in \mathbb{C} \backslash\{0\}:|\arg \lambda| \leq \phi\}, \phi \in\left(\frac{\pi}{2}, \pi\right)$.

(A:2) The resolvent operator is an almost-periodic mapping taking values in the space of linear operators on $\mathcal{H}: R\left(\omega_{0}, \mathcal{A}(\cdot)\right) \in A P(\mathbb{R} ; \mathcal{L}(\mathcal{H}))$ for $\omega_{0}$ as in $(\mathrm{A}: 1)$.

For a discussion of the above assumption we refer, for instance, to [15. The following result shows why in our main example assumption (A:2) is satisfied.

Lemma 3.1. A sufficient condition for (A:2) to hold, in case $\mathcal{A}(t)=\mathcal{A}+\mathcal{V}(t)$, is that $\mathcal{V}(\cdot) \in A P(\mathbb{R} ; \mathcal{L}(D(\mathcal{A}), \mathcal{H}))$.

Proof. Let $z(t)=R\left(\omega_{0}, \mathcal{A}(t)\right) x=\left(I-R\left(\omega_{0}, \mathcal{A}\right) \mathcal{V}(t)\right)^{-1} R\left(\omega_{0}, \mathcal{A}\right) x$ for given $x \in H$. Then it holds

$$
\left(I-R\left(\omega_{0}, \mathcal{A}\right) \mathcal{V}(t)\right) z(t)=\left(I-R\left(\omega_{0}, \mathcal{A}\right) \mathcal{V}(s)\right) z(s)
$$

and we get

$$
z(t)-z(s)=R\left(\omega_{0}, \mathcal{A}\right)[\mathcal{V}(t)-\mathcal{V}(s)] z(t)+R\left(\omega_{0}, \mathcal{A}\right) \mathcal{V}(s)[z(t)-z(s)]
$$

that is,

$$
z(t)-z(s)=R\left(\omega_{0}, \mathcal{A}(s)\right)[\mathcal{V}(t)-\mathcal{V}(s)] R\left(\omega_{0}, \mathcal{A}(t)\right) x .
$$

Fix $s, \varepsilon>0$ and $a$ and let $\tau=\tau_{\varepsilon}$ be the almost period of $B(\cdot)$ in $(a, a+\ell(\varepsilon))$; then

$$
\left\|R\left(\omega_{0}, \mathcal{A}(s+\tau)\right)-R\left(\omega_{0}, \mathcal{A}(s)\right)\right\|_{\mathcal{L}(\mathcal{H})} \leq K^{2} \varepsilon
$$

which shows that $R\left(\omega_{0}, \mathcal{A}(\cdot)\right)$ is almost periodic.

As a consequence of the above assumptions, we get that the solution of the linear nonautonomous problem is, in some sense, almost periodic. The following result is proved in [15, Proposition 4.4].

Lemma 3.2. Suppose that $\mathcal{A}(t)$ satisfies assumptions (A:1-2). Then the evolution family

$$
r \mapsto \mathcal{U}(t+r, s+r)
$$

is a $\mathcal{L}(\mathcal{H})$-valued almost periodic function for $t>s \in \mathbb{R}$. Further, given $\varepsilon>0$ and $h>0$, there exists $\ell(\varepsilon)>0$ such that for any interval of length $\ell(\varepsilon)$, there exists a number $\tau=\tau_{\varepsilon}$ in this interval such that

$$
\|\mathcal{U}(t+\tau, s+\tau)-\mathcal{U}(t, s)\|_{\mathcal{L}(\mathcal{H})} \leq \varepsilon e^{\frac{\omega_{V}}{2}|t-s|}
$$

for all $t>s \in \mathbb{R},|t-s|>h$.

We now consider the nonlinear coefficients $F: \mathbb{R} \times L^{2}(\Omega ; \mathcal{H}) \rightarrow L^{2}(\Omega ; \mathcal{H})$ and $G: \mathbb{R} \times L^{2}(\Omega, \mathcal{H}) \rightarrow L^{2}(\Omega, \mathcal{L}(\mathcal{H}))$.

(F) $F(t, \xi)$ is a jointly continuous mapping such that 
(a) for any compact $K \subset L^{2}(\Omega ; \mathcal{H})$ the process $t \mapsto F(t, \xi)$ is an $\mathcal{H}$-valued square-mean almost periodic process uniformly in $\xi \in K$, i.e., for any $\varepsilon>0$ there exists a real number $\ell=\ell(\varepsilon, K)>0$ such that for any $s \in \mathbb{R}$ we can find a point in the interval $(s, s+\ell(\varepsilon, K))$ with

$$
\sup _{t \in \mathbb{R}} \mathbb{E}\|F(t+\tau, \xi)-F(t, \xi)\|^{2}<\varepsilon
$$

for all $\xi \in K$;

(b) there exists a constant $K_{F}>0$ such that for all $\xi, \eta \in L^{2}(\Omega ; \mathcal{H})$ and for each $t \in \mathbb{R}$,

$$
\mathbb{E}\|F(t, \xi)-F(t, \eta)\|^{2} \leq K_{F} \mathbb{E}\|\xi-\eta\|^{2} .
$$

(G) $G(t, X)$ is a jointly continuous mapping such that

(a) for any compact $K^{\prime} \subset L^{2}(\Omega ; \mathcal{H})$ the process $t \mapsto G(t, \xi)$ is an $\mathcal{L}(\mathcal{H})$ valued square-mean almost periodic process uniformly in $\xi \in K^{\prime}$;

(b) there exists a constant $K_{G}>0$ such that for all $\xi, \eta \in L^{2}(\Omega ; \mathcal{H})$

$$
\mathbb{E}\|G(t, \xi)-G(t, \eta)\|_{\mathcal{L}(\mathcal{H})}^{2} \leq K_{G} \mathbb{E}\|\xi-\eta\|^{2}
$$

uniformly in $t \in \mathbb{R}$.

Remark 3.3. It is known (compare [2, Theorem 2.7]) that if $F: \mathbb{R} \times L^{2}(\Omega ; \mathcal{H}) \rightarrow$ $L^{2}(\Omega ; \mathcal{H})$ satisfies assumption $(F)$ above and $X(t)$ is a square-mean almost periodic process with values in $\mathcal{H}$ then the composition $t \mapsto F(t, X(t))$ is square-mean almost periodic as well.

The same remark applies for the composition $t \mapsto G(t, X(t))$ for any almostperiodic process $X(t)$ and $G: \mathbb{R} \times L^{2}(\Omega ; \mathcal{H}) \rightarrow L^{2}(\Omega ; \mathcal{L}(\mathcal{H}))$ which satisfies assumption $(G)$.

We can now proceed to present the main result of this section.

Theorem 3.4. Under Hypotheses (A:1-2), $(F)$ and $(G)$, assume that

$$
\frac{M_{1}^{2}}{\left|\omega_{V}\right|}\left(\frac{2 K_{F}}{\left|\omega_{V}\right|}+\nu_{2} K_{G}\right)<1
$$

where $\nu_{2}$ is the constant from assumption (2.9). Then there exists an unique squaremean almost periodic mild solution $X(t)$ of problem (1.3) which is explicitly given by the formula

$$
X(t)=\int_{-\infty}^{t} \mathcal{U}(t, r) F(r, X(r)) \mathrm{d} r+\int_{-\infty}^{t} \mathcal{U}(t, r) G(r, X(r)) \mathrm{d} L(r)
$$

for each $t \in \mathbb{R}$.

The proof of this theorem is rather technical so we provide a short outline for the sake of clearness.

It is known that the space $A P\left(\mathbb{R} ; L^{2}(\Omega ; \mathcal{H})\right)$ of $\mathcal{H}$-valued square-mean almost periodic processes is a Banach space when endowed with the norm

$$
\|X\|_{\infty}=\sup _{t \in \mathbb{R}}\left(\mathbb{E}\|X(t)\|^{2}\right)^{1 / 2} .
$$

Therefore, it is possible to apply on this space a fixed-point argument which will provide the existence of the solution. We introduce the mapping $\Lambda: A P\left(\mathbb{R} ; L^{2}(\Omega ; \mathcal{H})\right) \rightarrow$ 
$A P\left(\mathbb{R} ; L^{2}(\Omega ; \mathcal{H})\right)$ by

$$
\Lambda X(t):=\int_{-\infty}^{t} \mathcal{U}(t, r) F(r, X(r)) \mathrm{d} r+\int_{-\infty}^{t} \mathcal{U}(t, r) G(r, X(r)) \mathrm{d} L(r) .
$$

Our first task is to prove that this mapping is well-defined, compare Lemmas 3.5 and 3.6. Then, we prove that under condition (3.2), $\Lambda$ is a contraction, hence there exists a unique fixed point that is the solution for problem 1.3 .

Lemma 3.5. Under assumption (A:1-3) and $(F)$, let $X$ be a square-mean almost periodic process. Then

$$
\Psi(t)=\int_{-\infty}^{t} \mathcal{U}(t, r) F(r, X(r)) \mathrm{d} r
$$

is a square-mean almost periodic process with values in $\mathcal{H}$.

Proof. Let $\varepsilon>0$ and $h>0$; by assumption, $X$ is a square-mean almost periodic process, hence by Remark $3.3 F(r, X(r))$ is square-mean almost periodic as well. Moreover, by Lemma 3.2 we can choose $\ell(\varepsilon)>0$ such that any interval of length $\ell(\varepsilon)$ contains a number $\tau$ such that

$$
\sup _{r \in \mathbb{R}} \mathbb{E}\|F(r+\tau, X(r+\tau))-F(r, X(r))\|^{2} \leq \varepsilon
$$

and

$$
\|\mathcal{U}(t+\tau, s+\tau)-\mathcal{U}(t, s)\| \leq \varepsilon e^{\frac{\omega_{V}}{2}(t-s)}
$$

for all $t-s \geq h$.

For these values of $\varepsilon, h$ and $\tau$, we compute

$$
\begin{aligned}
\| \Psi(t+\tau)- & \Psi(t)\|=\| \int_{-\infty}^{t+\tau} \mathcal{U}(t+\tau, r) F(r, X(r)) \mathrm{d} r-\int_{-\infty}^{t} \mathcal{U}(t, r) F(r, X(r)) \mathrm{d} r \| \\
\leq & \int_{t-h}^{t}\|\mathcal{U}(t+\tau, r+\tau)-\mathcal{U}(t, r)\|_{\mathcal{L}(\mathcal{H})}\|F(r, X(r))\| \mathrm{d} r \\
& +\int_{-\infty}^{t-h}\|\mathcal{U}(t+\tau, r+\tau)-\mathcal{U}(t, r)\|_{\mathcal{L}(\mathcal{H})}\|F(r, X(r))\| \mathrm{d} r \\
& +\int_{-\infty}^{t}\|\mathcal{U}(t+\tau, r+\tau)\|_{\mathcal{L}(\mathcal{H})}\|F(r+\tau, X(r+\tau))-F(r, X(r))\| \mathrm{d} r
\end{aligned}
$$

The above computation thus leads to

$$
\begin{aligned}
& \mathbb{E}\|\Psi(t+\tau)-\Psi(t)\|^{2} \\
& \leq 3 \mathbb{E}\left(\int_{t-h}^{t}\|\mathcal{U}(t+\tau, r+\tau)-\mathcal{U}(t, r)\|_{\mathcal{L}(\mathcal{H})}\|F(r, X(r))\| \mathrm{d} r\right)^{2} \\
& \quad+3 \mathbb{E}\left(\int_{-\infty}^{t-h}\|\mathcal{U}(t+\tau, r+\tau)-\mathcal{U}(t, r)\|_{\mathcal{L}(\mathcal{H})}\|F(r, X(r))\| \mathrm{d} r\right)^{2} \\
& \quad+3 \mathbb{E}\left(\int_{-\infty}^{t}\|\mathcal{U}(t+\tau, r+\tau)\|_{\mathcal{L}(\mathcal{H})}\|F(r+\tau, X(r+\tau))-F(r, X(r))\| \mathrm{d} r\right)^{2} \\
& =I_{1}+I_{2}+I_{3} .
\end{aligned}
$$


The first term is bounded as follows: since $\|\mathcal{U}(t, s)\|_{\mathcal{L}(\mathcal{H})} \leq M_{1} e^{\omega_{V}(t-s)}$, it follows by Hölder inequality that

$$
I_{1} \leq 3\left(\int_{t-h}^{t} 2 M_{1} e^{\omega_{V}(t-r)} \mathrm{d} r\right) \mathbb{E}\left(\int_{t-h}^{t} 2 M_{1} e^{\omega_{V}(t-r)}\|F(r, X(r))\|^{2} \mathrm{~d} r\right)
$$

and the boundedness of the square-mean almost periodic process $F(r, X(r))$ implies that there is $N_{F}>0$ such that $\sup _{t \in \mathbb{R}} \mathbb{E}\|F(r, X(r))\|^{2} \leq N_{F}$ (see Lemma 1.3), thus

$$
I_{1} \leq 3 N_{F}\left[\frac{2 M}{\omega_{V}}\left(1-e^{\omega_{V} h}\right)\right]^{2} .
$$

Now we use 3.1 in the second integral (here $|t-r| \geq h$ ), again with the inequality $\sup _{t \in \mathbb{R}} \mathbb{E}\|F(r, X(r))\|^{2} \leq N_{F}$ and Hölder inequality, to get

$$
I_{2} \leq 3 \mathbb{E}\left(\int_{-\infty}^{t-h} \varepsilon e^{\frac{\omega_{V}}{2}(t-r)}\|F(r, X(r))\| \mathrm{d} r\right)^{2} \leq 3 N_{F}\left[\frac{2 \varepsilon}{\omega_{V}} e^{\frac{\omega_{V}}{2}} h\right]^{2}
$$

Finally, since $F(r, X(r))$ is square-mean almost periodic with $\varepsilon$-almost period $\tau$, it holds that

$I_{3} \leq 3\left(\int_{-\infty}^{t} M_{1} e^{\omega_{V}(t-r)} \mathrm{d} r\right)^{2} \sup _{r \in \mathbb{R}} \mathbb{E}\|F(r+\tau, X(r+\tau))-F(r, X(r))\|^{2}=3 \varepsilon \frac{M_{1}^{2}}{\omega_{V}^{2}}$

Choose $h$ such that $\left(1-e^{\omega_{V} h}\right)=\varepsilon$; letting

$$
\varepsilon^{\prime}=12 N_{F} \frac{M^{2}}{\omega_{V}^{2}} \varepsilon^{2}+12 N_{F} \frac{\varepsilon^{2}}{\omega_{V}^{2}}(1-\varepsilon)+3 \varepsilon \frac{M^{2}}{\omega_{V}^{2}}
$$

we have thus proved that $\Psi$ is a square-mean almost periodic process with $\varepsilon^{\prime}$-almost period $\tau$.

Lemma 3.6. Under assumption (A:1-2) and $(G)$, let $X$ be a square-mean almost periodic process. Then

$$
\Phi(t)=\int_{-\infty}^{t} \mathcal{U}(t, r) G(r, X(r)) \mathrm{d} L(r)
$$

is a square-mean almost periodic process with values in $\mathcal{H}$.

Proof. Let $\varepsilon>0$ and $h>0$; by assumption, $X$ is a square-mean almost periodic process, hence by Remark $3.3 G(r, X(r))$ is square-mean almost periodic as well. Moreover, by Lemma 3.2 we can choose $\ell(\varepsilon)>0$ such that any interval of length $\ell(\varepsilon)$ contains a number $\tau$ such that

$$
\sup _{r \in \mathbb{R}} \mathbb{E}\|G(r+\tau, X(r+\tau))-G(r, X(r))\|_{\mathcal{L}(\mathcal{H})}^{2} \leq \varepsilon
$$

and

$$
\|\mathcal{U}(t+\tau, s+\tau)-\mathcal{U}(t, s)\| \leq \varepsilon e^{\frac{\omega_{V}}{2}(t-s)}
$$

for all $t-s \geq h$.

In order to avoid difficulties when we change variables in stochastic integrals, we define $\tilde{L}(s):=L(s+t)-L(t)$ for each $s \in \mathbb{R}$ and fixed $t$. Note that $\tilde{L}$ is also a Lévy process and has the same distribution as $L$. 
Now we compute

$$
\begin{aligned}
\mathbb{E} \| \Phi(t+\tau) & -\Phi(t) \|^{2} \\
= & \mathbb{E}\left\|\int_{-\infty}^{t+\tau} \mathcal{U}(t+\tau, r) G(r, X(r)) \mathrm{d} L(r)-\int_{-\infty}^{t} \mathcal{U}(t, r) G(r, X(r)) \mathrm{d} L(r)\right\|^{2} \\
= & \mathbb{E} \| \int_{0}^{+\infty} \mathcal{U}(t+\tau, t+\tau-s) G(t+\tau-s, X(t+\tau-s)) \mathrm{d} \tilde{L}(s) \\
& \quad-\int_{0}^{+\infty} \mathcal{U}(t, t-s) G(t-s, X(t-s)) \mathrm{d} \tilde{L}(s) \|^{2} \\
\leq 3 \mathbb{E} \| \int_{0}^{+\infty} & \mathcal{U}(t+\tau, t+\tau-s)[G(t+\tau-s, X(t+\tau-s))-G(t-s, X(t-s))] \mathrm{d} \tilde{L}(s) \|^{2} \\
& +3 \mathbb{E}\left\|\int_{h}^{+\infty}[\mathcal{U}(t+\tau, t+\tau-s)-\mathcal{U}(t, t-s)] G(t-s, X(t-s)) \mathrm{d} \tilde{L}(s)\right\|^{2} \\
& +3 \mathbb{E}\left\|\int_{0}^{h}[\mathcal{U}(t+\tau, t+\tau-s)-\mathcal{U}(t, t-s)] G(t-s, X(t-s)) \mathrm{d} \tilde{L}(s)\right\|^{2}
\end{aligned}
$$

Using the classical isometry for Poisson integrals and denoting by $\nu_{2}$ the finite second order moment of $\nu$ (recall condition (2.9p), we obtain

$$
\begin{aligned}
\mathbb{E}\|\Phi(t+\tau)-\Phi(t)\|^{2} \leq 3 \nu_{2} \int_{0}^{+\infty}\|\mathcal{U}(t+\tau, t+\tau-s)\|_{\mathcal{L}(\mathcal{H})}^{2} \times & \times \mathbb{E}\|G(t+\tau-s, X(t+\tau-s))-G(t-s, X(t-s))\|_{\mathcal{L}(\mathcal{H})}^{2} \mathrm{~d} s \\
& +3 \nu_{2} \int_{h}^{+\infty}\|\mathcal{U}(t+\tau, t+\tau-s)-\mathcal{U}(t, t-s)\|_{\mathcal{L}(\mathcal{H})}^{2} \mathbb{E}\|G(t-s, X(t-s))\|_{\mathcal{L}(\mathcal{H})}^{2} \mathrm{~d} s \\
& +3 \nu_{2} \int_{0}^{h}\|\mathcal{U}(t+\tau, t+\tau-s)-\mathcal{U}(t, t-s)\|_{\mathcal{L}(\mathcal{H})}^{2} \mathbb{E}\|G(t-s, X(t-s))\|_{\mathcal{L}(\mathcal{H})}^{2} \mathrm{~d} s \\
\leq & 3 M_{1}^{2} \nu_{2}\left(\int_{0}^{+\infty} e^{2 \omega_{V} s} \mathrm{~d} s\right) \sup _{r \in \mathbb{R}} \mathbb{E}\|G(r+\tau, X(r+\tau))-G(r, X(r))\|_{\mathcal{L}(\mathcal{H})}^{2} \\
& +3 M_{1}^{2} \varepsilon^{2} \nu_{2}\left(\int_{h}^{+\infty} e^{\omega_{V} s} \mathrm{~d} s\right) \sup _{r \in \mathbb{R}} \mathbb{E}\|G(r, X(r))\|_{\mathcal{L}(\mathcal{H})}^{2} \\
& +6 M_{1}^{2} \nu_{2}\left(\int_{0}^{h} e^{2 \omega_{V} s} \mathrm{~d} s\right) \sup _{r \in \mathbb{R}} \mathbb{E}\|G(r, X(r))\|_{\mathcal{L}(\mathcal{H})}^{2} \\
\leq & 3 \frac{\nu_{2}}{\left|\omega_{V}\right|}\left[\varepsilon \frac{M_{1}^{2}}{2}+\varepsilon^{2} N_{G} e^{\omega_{V} h}+\varepsilon \frac{2 M_{1}^{2} N_{G}}{2}\left(1-e^{2 \omega_{V} h}\right)\right]
\end{aligned}
$$

which implies, choosing $h$ in such a way $1-e^{2 \omega_{V} h}=\varepsilon$, that

$$
\sup _{t \in \mathbb{R}} \mathbb{E}\|\Phi(t+\tau)-\Phi(t)\|^{2} \leq 3 \frac{\nu_{2}}{\left|\omega_{V}\right|}\left[\varepsilon \frac{M_{1}^{2}}{2}+\varepsilon^{2} \sqrt{1-\varepsilon} N_{G}+\varepsilon^{2} \frac{2 M_{1}^{2} N_{G}}{2}\right]=: \varepsilon^{\prime}
$$

i.e. $\Phi(t)$ is square-mean almost periodic with $\varepsilon^{\prime}$-almost period $\tau$.

Lemma 3.7. The mapping $\Lambda$ defined in 3.4 is a contraction on the space of almost periodic processes $A P\left(\mathbb{R} ; L^{2}(\Omega ; \mathcal{H})\right)$. 
Proof. Let us fix $X, Y \in A P\left(\mathbb{R} ; L^{2}(\Omega ; \mathcal{H})\right)$; then we get

$$
\begin{aligned}
\mathbb{E}\|\Lambda X(t)-\Lambda Y(t)\|^{2} \leq & 2 \mathbb{E}\left(\int_{-\infty}^{t}\|\mathcal{U}(t, r)\|_{\mathcal{L}(\mathcal{H})}\|F(r, X(r))-F(r, Y(r))\| \mathrm{d} r\right)^{2} \\
& +2 \mathbb{E}\left\|\int_{-\infty}^{t} \mathcal{U}(t, r)[G(r, X(r))-G(r, Y(r))] \mathrm{d} L(r)\right\|^{2} \\
\leq & 2 \mathbb{E}\left(\int_{-\infty}^{t}\|\mathcal{U}(t, r)\|_{\mathcal{L}(\mathcal{H})}\|F(r, X(r))-F(r, Y(r))\| \mathrm{d} r\right)^{2} \\
& +2 \nu_{2} \int_{-\infty}^{t}\|\mathcal{U}(t, r)\|_{\mathcal{L}(\mathcal{H})}^{2} \mathbb{E}\|G(r, X(r))-G(r, Y(r))\|_{\mathcal{L}(\mathcal{H})}^{2} \mathrm{~d} r
\end{aligned}
$$

where we have used the isometry for the Lévy integral in the last term; recalling the Lipschitz estimates on $F$ and $G$, the growth bound on $\mathcal{U}(t, s)$ we obtain

$$
\begin{aligned}
\mathbb{E} \| \Lambda X(t)- & \Lambda Y(t) \|^{2} \\
\leq & 2 M_{1}^{2}\left(\int_{-\infty}^{t} e^{\omega_{V}(t-r)} \mathrm{d} r\right)\left(\int_{-\infty}^{t} e^{\omega_{V}(t-r)} \mathbb{E}\|F(r, X(r))-F(r, Y(r))\|^{2} \mathrm{~d} r\right) \\
& +2 \nu_{2} M_{1}^{2} K_{G} \int_{-\infty}^{t} e^{2 \omega_{V}(t-r)} \mathbb{E}\|X(r)-Y(r)\|^{2} \mathrm{~d} r \\
\leq & 2 M_{1}^{2} K_{F}\left(\int_{-\infty}^{t} e^{\omega_{V}(t-r)} \mathrm{d} r\right)\left(\int_{-\infty}^{t} e^{\omega_{V}(t-r)} \mathbb{E}\|X(r)-Y(r)\|^{2} \mathrm{~d} r\right) \\
& +2 \nu_{2} M_{1}^{2} K_{G} \int_{-\infty}^{t} e^{2 \omega_{V}(t-r)} \mathbb{E}\|X(r)-Y(r)\|^{2} \mathrm{~d} r \\
\leq & 2 M_{1}^{2} K_{F}\left(\int_{-\infty}^{t} e^{\omega_{V}(t-r)} \mathrm{d} r\right)^{2} \sup _{t \in \mathbb{R}} \mathbb{E}\|X(t)-Y(t)\|^{2} \\
& +2 \nu_{2} M_{1}^{2} K_{G}\left(\int_{-\infty}^{t} e^{2 \omega_{V}(t-r)} \mathrm{d} r\right) \sup _{t \in \mathbb{R}} \mathbb{E}\|X(t)-Y(t)\|^{2} \\
= & \frac{M_{1}^{2}}{\left|\omega_{V}\right|}\left(\frac{2 K_{F}}{\left|\omega_{V}\right|}+\nu_{2} K_{G}\right)\|X-Y\|_{\infty}^{2} .
\end{aligned}
$$

Thus it follows that

$$
\|\Lambda X-\Lambda Y\|_{\infty}^{2} \leq \frac{M^{2}}{\left|\omega_{V}\right|}\left(\frac{2 K_{F}}{\left|\omega_{V}\right|}+\nu_{2} K_{G}\right)\|X-Y\|_{\infty} .
$$

Consequently, if condition 3.2 holds, we get that the mapping $\Lambda$ defined in (3.4) has a unique fixed point, which obviously is the unique almost periodic mild solution of $(3.3)$.

\section{REFERENCES}

[1] P. H. Bezandry and T. Diagana. Existence of almost periodic solutions to some stochastic differential equations. Appl. Anal., 86(7):819-827, 2007.

[2] P. H. Bezandry and T. Diagana. Square-mean almost periodic solutions nonautonomous stochastic differential equations. Electron. J. Differential Equations, pages No. 117, 10 pp. (electronic), 2007.

[3] S. Bonaccorsi, C. Marinelli, and G. Ziglio. Stochastic FitzHugh-Nagumo equations on networks with impulsive noise. Electron. J. Probab., 13:no. 49, 1362-1379, 2008.

[4] S. Bonaccorsi and D. Mugnolo. Existence of strong solutions for neuronal network dynamics driven by fractional Brownian motions. Stochastics and Dynamics, 10(3):441-464, 2010. 
[5] A. Chen and J. Cao. Almost periodic solution of shunting inhibitory CNNs with delays. Physics Letters A, 298(2-3):161 - 170, 2002.

[6] L. O. Chua and L. Yang. Cellular neural networks: applications. IEEE Trans. Circuits and Systems, 35(10):1273-1290, 1988.

[7] L. O. Chua and L. Yang. Cellular neural networks: theory. IEEE Trans. Circuits and Systems, 35(10):1257-1272, 1988

[8] C. Corduneanu. Almost Periodic Oscillations and Waves. Springer Science+Business Media, 2009.

[9] G. Da Prato and C. Tudor. Periodic and almost periodic solutions for semilinear stochastic equations. Stochastic Anal. Appl., 13(1):13-33, 1995.

[10] A. M. Fink. Almost periodic differential equations. Lecture Notes in Mathematics, Vol. 377. Springer-Verlag, Berlin, 1974.

[11] D. Henry. Geometric theory of semilinear parabolic equations, volume 840 of Lecture Notes in Mathematics. Springer-Verlag, Berlin, 1981.

[12] J. J. Hopfield. Neural networks and physical systems with emergent collective computational abilities. Proc. Nat. Acad. Sci. U.S.A., 79(8):2554-2558, 1982.

[13] G. Kallianpur and J. Xiong. Diffusion approximation of nuclear space-valued stochasticdifferential equations driven by Poisson random measures. Ann. Appl. Probab., 5(2):493-517, 1995.

[14] G. Major, J. D. Evans, and J. J. Jack. Solutions for transients in arbitrarily branching cables: I. voltage recording with a somatic shunt. Biophys. J, 65:423-449, 1993.

[15] L. Maniar and R. Schnaubelt. Almost periodicity of inhomogeneous parabolic evolution equations. In Evolution equations, volume 234 of Lecture Notes in Pure and Appl. Math., pages 299-318. Dekker, New York, 2003.

[16] D. Mugnolo and S. Romanelli. Dynamic and generalized Wentzell node conditions for network equations. Math. Methods Appl. Sci., 30(6):681-706, 2007.

[17] C. Tudor. Almost periodic solutions of affine stochastic evolution equations. Stochastics Stochastics Rep., 38(4):251-266, 1992.

Stefano BonacCorsi

Dipartimento di Matematica, Università di Trento, via Sommarive 14, 38123 Povo (Trento), ITALIA

E-mail address: stefano.bonaccorsi@unitn.it

Giacomo Ziglio

Dipartimento di Matematica, Università di Trento, via Sommarive 14, 38123 Povo (Trento), ITALIA

E-mail address: giacomo.ziglio@gmail.com 